\title{
The effects of lead stimulus and reflex stimulus modality on modulation of the blink reflex at very short, short, and long lead intervals
}

\author{
DAVID L. NEUMANN \\ Griffith University, Queensland, Australia \\ and \\ OTTMAR V. LIPP and NATALIE R. PRETORIUS \\ University of Queensland, Queensland, Australia
}

\begin{abstract}
The blink reflex is modulated if a weak lead stimulus precedes the blink-eliciting stimulus. In two experiments, we examined the effects of the sensory modality of the lead and blink-eliciting stimuli on blink modulation. Acoustic, visual, or tactile lead stimuli were followed by an acoustic (Experiment 1) or an electrotactile (Experiment 2) blink-eliciting stimulus at lead intervals of -30, 0, 30, 60, 120, 240, 360 , and 4,500 msec. The inhibition of blink magnitude at the short (60- to 360-msec) lead intervals and the facilitation of blink magnitude at the long (4,500-msec) lead interval observed for each lead stimulus modality was relatively unaffected by the blink-eliciting stimulus modality. The facilitation of blink magnitude at the very short ( -30 - to 30 -msec) lead intervals was dependent on the combination of the lead and the blink-eliciting stimulus modalities. Modality specific and nonspecific processes operate at different levels of perceptual processing.
\end{abstract}

The blink reflex is elicited if an individual is presented with a sudden and moderately intense stimulus. The magnitude of the blink reflex can be modulated by the nature of the blink-eliciting stimulus, by a stimulus that precedes it (the lead stimulus), and by the time interval between the lead stimulus and the blink-eliciting stimulus (the lead interval). As with other authors (e.g., Blumenthal, 1999; Filion, Dawson, \& Schell, 1998; Lipp, Blumenthal, \& Adam, 2001 ), lead intervals of $60 \mathrm{msec}$ or less are termed very short, lead intervals greater than $60 \mathrm{msec}$ and up to $500 \mathrm{msec}$ are termed short, and lead intervals longer than $500 \mathrm{msec}$ are termed long. ${ }^{1}$ In addition, the lead stimulus may be discrete, or it may be sustained throughout the lead interval. Discrete and sustained lead stimuli do not appear to influence blink modulation differentially at very short or short lead intervals (Graham \& Murray, 1977) but may exert different influences on modulation at long lead intervals (Graham, Putnam, \& Leavitt, 1975). Blink reflex modulation is of interest because it is thought to reflect the

This work was supported by Grant A10027218 from the Australian Research Council. Part of this work was completed while the first author was at the School of Psychology, University of Queensland. Thanks to Mathew T. Martin-Iverson for a helpful discussion concerning the results reported in this article. Correspondence should be addressed to O. V. Lipp, School of Psychology, University of Queensland, Queensland 4072, Australia (e-mail: o.lipp@ psy.uq.edu.au)or D. L. Neumann, School of Applied Psychology, Griffith University (Gold Coast Campus), PMB 50 Gold Coast Mail Centre, Queensland 9726, Australia (e-mail: d.neumann@griffith.edu.au). flow of information in the human processing system. As the lead interval shortens, blink modulation predominantly reflects automatic preattentive processes. Modulation at longer lead intervals, by contrast, tends to reflect the effects of higher cognitive function (Filion et al., 1998). However, these rules are by no means exclusive. Attentional and emotional processes may modulate blinks at very short and short lead intervals (Aitken, Siddle, \& Lipp, 1999; Lipp et al., 2001), and perceptual processes may influence long lead interval blink modulation (Graham, 1975).

A significant feature of the blink reflex is that it can be elicited by stimuli from various sensory modalities, such as acoustic, visual, or tactile (Berg \& Balaban, 1999). In research on blink reflex modulation, the role of stimulus modality has not yet been examined in detail. In other research domains, however, there has been an increasing number of studies in which similarities and differences across the stimulus modalities and also the cross-modal links in perception and attention have been examined (e.g., Dell'Acqua, Turatto, \& Jolicœur, 2001; Driver \& Spence, 2001; Tekok-Kilic, Shucard, \& Shucard, 2001). Such research emphasizes the multimodal nature of the everyday situation and can seek to answer many interesting questions that are often excluded when only one stimulus modality is examined (Driver \& Spence, 2001). The aim of the present study was to examine the effects of stimulus modality in order to further our understanding of the perceptual and attentional processes that are reflected in very short, short, and long lead interval blink modulation. 
At very short lead intervals, the blink reflex tends to be facilitated, relative to blink-eliciting stimulus-alone trials, when the lead stimulus and the blink-eliciting stimulus are from different sensory modalities. Previous studies have combined acoustic or visual lead stimuli with noise (Aitken et al., 1999; Lipp et al., 2001), air puff (Flaten \& Blumenthal, 1996), or electrical stimulation of the trigeminal nerve (Boelhouwer, Teurlings, \& Brunia, 1991; Sarno, Blumenthal, \& Boelhouwer, 1997; Schmolesky, Boelhouwer, \& Blumenthal, 1996) as the blink-eliciting stimulus. Boelhouwer et al. (1991) interpreted very short lead interval facilitation as reflecting temporal summation. It was suggested that the facial motor nucleus activity generated by the lead stimulus and the blink-eliciting stimulus will overlap and sum as the interval between the two stimuli decreases. However, when stimuli from different sensory modalities are presented, the final summation will depend on both the speed of transmission in the sensory pathway of the lead stimulus and the blink-eliciting stimulus and the lead interval between them (Rimpel, Geyer, \& Hopf, 1982). The investigation of very short lead interval facilitation may prove useful in determining the convergence of and the conduction velocities within sensory pathways (Sarno et al., 1997). In the present study, we examined very short lead interval facilitation under a wider range of lead stimulus and blink-eliciting stimulus modalities than had been used previously.

Blink inhibition occurs at short lead intervals in a variety of lead stimulus and blink-eliciting stimulus arrangements. Graham (1975) has suggested that short lead interval inhibition reflects an automatic transient detection process initiated by lead stimulus presentation. This transient detection process serves to protect the processing of the lead stimulus from interruption by the blink-eliciting stimulus and also serves to initiate processes that lead to the identification of the lead stimulus. An inspection of previous research suggests that the time course of short lead interval inhibition of acoustic blinks differs between different lead stimulus modalities. For acoustic lead stimuli, maximal inhibition occurs at approximately 100$150 \mathrm{msec}$ (Graham et al., 1975), with inhibition decreasing at lead intervals outside this range (Graham, 1975). Tactile lead stimuli tend to delay the occurrence of maximal inhibition of acoustic blinks to lead intervals of 150-250 msec (Blumenthal \& Tolomeo, 1989), whereas inhibition of acoustic blinks is maximal at lead intervals of $240 \mathrm{msec}$ for visual lead stimuli (Aitken et al., 1999; Lipp et al., 2001). It is possible, however, that the differences in the time course of blink inhibition across the different studies may reflect factors other than stimulus modality, such as lead stimulus intensity. In the present experiments, we thus examined short lead interval blink inhibition across a range of lead intervals in different stimulus modalities that were presented at the same intensity.

Lipp, Siddle, and Dall (2000b) examined the effect of stimulus modality on long lead interval blink modulation and found facilitation of the acoustic blink reflex. Moreover, the facilitation was larger for acoustic lead stimuli than for visual and tactile lead stimuli, whereas there was little difference between the visual and the tactile lead stimulus conditions. The differences across lead stimulus modalities may reflect the fact that Lipp et al.'s (2000b) study employed sustained lead stimuli that were present throughout the lead interval. Graham et al. (1975) reported larger facilitation of the acoustic blink reflex at long lead intervals with a sustained than with a discrete acoustic lead stimulus. Graham (1975) interpreted the facilitation following discrete lead stimuli as reflecting an orientingattentional process. The participants were thought to have attended to the lead stimulus presentation because it served as a cue for an anticipated blink-eliciting stimulus. It was suggested that long lead interval facilitation for sustained lead stimuli reflects a summation of the orientingattentional process and a classical activation effect caused by the sustained portion of the acoustic lead stimulus. An alternative interpretation of the differences in blink facilitation across different lead stimulus modalities may be that it reflects modality-specific attentional processes. Putnam (1990) suggested that blink facilitation will occur when there is a match between the modalities of the lead and the blink-eliciting stimuli, but not when there is a modality mismatch.

The aim of the present research was to examine the effects of lead stimulus and blink-eliciting stimulus modality on blink reflex modulation. Sustained lead stimuli from the acoustic, visual, and tactile stimulus modalities were employed in a passive attention task. Due to the effects that lead stimulus intensity can have on blink modulation (e.g., Schmolesky et al., 1996), the lead stimuli were matched on psychophysical intensity. A wide range of lead intervals was examined $(-30,0,30,60,120,240$, 360 , and $4,500 \mathrm{msec}$ ) to enable the simultaneous exploration of very short, short, and long lead interval blink modulation effects. We used an acoustic blink-eliciting stimulus in Experiment 1 because it has proven to be the most reliable method by which to elicit blinks and has been most commonly used in previous research. It was hypothesized that very short lead interval facilitation would be found for visual and tactile lead stimuli and not for acoustic lead stimuli, consistent with the observation of cross-modal effects in previous experiments (Blumenthal, 1999). The development of short lead interval inhibition was hypothesized to occur earlier for acoustic lead stimuli than for visual and tactile lead stimuli. Finally, long lead interval facilitation was expected to be larger for acoustic lead stimuli than for visual and tactile lead stimuli (Lipp et al., 2000b).

\section{EXPERIMENT 1}

\section{Method}

Participants. Eleven male and 66 female students from the University of Queensland participated after providing informed consent. The participants received course credit or AUS $\$ 10$ for their participation. The data from 4 male and 18 female participants were excluded due to excessive missing blink reflex data, and a further 2 female participants were excluded due to extreme values in the blink reflex data. The final sample had a mean age of 19.2 years $(S D=$ 
4.44). Upon arrival at the laboratory, the participants were randomly allocated to one of the three groups in such a way that the final sample sizes and the male-to-female ratios were 3:14 in the acoustic lead stimulus group, 1:17 in the visual lead stimulus group, and 3:15 in the tactile lead stimulus group. The groups did not differ in sex distribution $\left[\chi^{2}(2)=1.4\right]$ or age $[F(2,50)=0.65$, all $p \mathrm{~s}>.05]$.

Apparatus. The blink reflex was measured by recording the electromyographic (EMG) activity of the orbicularis oculi muscle with a pair of $\mathrm{Ag} / \mathrm{AgCl}$ domed electrodes ( $4 \mathrm{~mm}$ in diameter) filled with a standard electrolyte (Surgicon E10). One electrode was placed under the pupil of the left eye, and the second was placed $1 \mathrm{~cm}$ lateral. The ground electrode was strapped to the inside of the left forearm. A Grass 7P3C AC preamplifier amplified the raw EMG signal, using a 0.5-amplitude highpass cutoff of $10 \mathrm{~Hz}$ and a low-pass cutoff of $3000 \mathrm{~Hz}$. The raw EMG was displayed on a Grass 7D polygraph (calibration: $100 \mu \mathrm{V} / \mathrm{cm}$ pen deflection), using a paper speed of $2.5 \mathrm{~mm} / \mathrm{sec}$, and was digitized and sampled on line, using a sampling rate of $1000 \mathrm{~Hz}$, with an IBMcompatible (486) computer. The sample window was $100 \mathrm{msec}$ prior to and $400 \mathrm{msec}$ after the blink-eliciting stimulus onset. The computer that sampled the EMG also controlled the stimulus sequences, stimulus duration, and intertrial intervals.

The blink-eliciting stimulus was a $105-\mathrm{dB}(\mathrm{A})$ burst of white noise presented with a duration of $50 \mathrm{msec}$ and an instantaneous rise time. The nature of the lead stimulus varied among the groups. In the acoustic lead stimulus group, a $1000-\mathrm{Hz}$ tone, presented at an intensity of $70 \mathrm{~dB}(\mathrm{~A})$, and an instantaneous rise time were used. All the auditory stimuli were generated by a custom-built tone generator and presented via stereophonic headphones (Sennheisser HD25-1). In the visual lead stimulus group, a picture of a black rectangle on a red background was projected by a Leica Pradovit P600 slide projector fitted with a Gerbrand G1166(S) tachistoscopic shutter through a screen set into the wall of the experimental room. The screen was located $140 \mathrm{~cm}$ from the participant and subtended $6.13^{\circ} \times 8.17^{\circ}$ of visual angle. In the tactile lead stimulus group, the vibration unit of a Mowat sensor was placed in a $2 \times 3 \times 4 \mathrm{~cm}$ metal housing and was taped to the second phalanx of the middle finger of the participant's nonpreferred hand. The vibration unit was driven by a custom-built power supply at a frequency of $50 \mathrm{~Hz}$. When activated, the unit produced a mild vibratory stimulation to the participant's finger. In all the groups, the lead stimulus was presented for a constant duration of $5 \mathrm{sec}$.

Prior to the experiment, pilot work was conducted to match the lead stimuli presented in each sensory modality on intensity. It was deemed that an individual's self-reported perceived intensity of a stimulus was the most appropriate measure of intensity. Initially, the intensity of the stimulation produced by the vibration unit was matched with the tone lead stimulus. In this procedure, the vibration stimulus was kept constant, and the intensity of the tone was varied in an upward and downward staircase procedure. The participants were presented with 5-sec presentations of each stimulus, separated by a 5 -sec interval. The participants were asked whether the intensity of the tone should be increased (or decreased) in order to match the intensity of the vibration and tone stimuli. If the participant requested that the tone should be increased (or decreased), the tone intensity was changed in steps of $5 \mathrm{~dB}(\mathrm{~A})$. The final intensity level for the tone was the average of the two procedures. The visual lead stimulus was selected on the basis that, in previous research, the stimulus had produced electrodermal responses equivalent to those for the vibration lead stimulus. This finding was taken as an indication that the stimuli did not differ in salience or intensity.

Procedure. The participants were seated in a chair opposite the projection screen in a darkened room, and the experiment was monitored from an adjoining room. Following electrode placement, the participants received one or more presentations of the blink-eliciting stimulus, to check the blink reflex recordings. The participants were next told that they would receive presentations of a tone/picture/vibration and would hear a noise through the headphones and that they should pay attention to all the stimuli because they might be asked questions about them at the end of the experiment. The participants were further told to maintain their gaze toward the projection screen in front of them and to minimize body movements. Following a 2min rest period, during which the participants sat quietly with their eyes open, the experiment began. The participants were presented with two blocks of trials, each block consisting of two presentations of the lead stimulus and the blink-eliciting stimulus at the following lead intervals: $-30,0,30,60,120,240,360$, and 4,500 msec (a negative lead interval indicates that the blink-eliciting stimulus was presented before the onset of the lead stimulus). Although a number of different late lead intervals could have been used, it was decided to use a lead interval of $4,500 \mathrm{msec}$, since this represented a lead interval duration typical in previous research (e.g., Lipp, Siddel, \& Dall, 2000a, 2000b; Putnam, 1990). In addition, the relatively small number of trials for each lead interval is typical in research in which blink reflex modulation is examined (e.g., Aitken et al., 1999), since the blink reflex decreases in magnitude (i.e., habituates) following repeated presentations of the blink-eliciting stimulus. The number of trials was thus limited in an effort to reduce the impact of habituation effects. Each block also contained eight presentations of the blink-eliciting stimulus alone. Trial order was randomized, with the restriction that two presentations of the blink-eliciting stimulus alone could not occur in succession. In addition, at the completion of the two blocks of trials, all the participants were presented with the tone lead stimulus followed by the blink-eliciting stimulus at a lead interval of $120 \mathrm{msec}$. However, since statistical analysis did not yield any differences between the groups for this trial, it will not be considered further. Throughout the experiment, the intertrial intervals varied at random between 12,15 , and $18 \mathrm{sec}$, onset to onset.

Scoring, response definition, and statistical analysis. The digitized EMG activity was rectified off line and was filtered and integrated with a Butterworth low-pass filter, using a time constant of $80 \mathrm{msec}$. The integrated signal was used to measure blink reflex magnitude. Blink reflex magnitude was measured as the difference between the maximum of the integrated curve within $200 \mathrm{msec}$ after blink-eliciting stimulus onset and the value of the integrated response curve at reflex onset. The onset of the blink reflex was defined as the point at which $10 \%$ of the maximum slope was reached. Blink latency, defined as the time between the onset of the blinkeliciting stimulus and the onset of the response, was also scored. Statistical analyses of response latency did not yield any systematic differences between groups and will not be reported. Trials were discarded from the data set if the baseline EMG was not stable within $100 \mathrm{msec}$ before the blink-eliciting stimulus or if the onset of the response was not within 20-70 msec after the onset of the blink-eliciting stimulus. On trials in which no response was detectable, magnitude was scored as zero.

The complete data set from a participant was discarded if the participant showed nonresponsiveness to the blink-eliciting stimulus. Nonresponsiveness was defined as having eight or more blinkeliciting stimulus-alone trials scored as missing or as having zero magnitude. With this criterion, 8 participants from the acoustic and tactile lead stimulus groups and 6 participants from the visual lead stimulus group were excluded. The number of participants that were excluded in the present study $(28.6 \%)$ is somewhat higher than that in previous experiments (e.g., 7.9\% in Aitken et al., 1999), largely due to the relatively large number of trials in the present experiment. Two further participants were excluded from the analyses following data screening, due to extreme values on multiple dependent variables.

Blink magnitudes on the blink-eliciting stimulus-alone trials and on the lead stimulus + blink-eliciting stimulus trials were averaged across the experiment. Missing values were replaced with mean values for the respective variable and group. This resulted in less than $1 \%$ of the data being replaced. Baseline level of responsiveness to the blink-eliciting stimulus was compared between groups, using a one-way analysis of variance (ANOVA). Blink modulation at each 
lead interval was examined by calculating change scores, using the formula (lead stimulus + blink-eliciting stimulus trial - blink-eliciting stimulus-alone trial)/blink-eliciting stimulus-alone trial $* 100$. A negative change score indicates inhibition, whereas a positive change score indicates facilitation. The $95 \%$ confidence intervals of the means were inspected to determine whether blink modulation was significantly different from zero. Modulation was reported as significant if zero was outside the confidence interval. Blink modulation at each lead interval was compared between groups with a $3 \times 8$ (group $\times$ lead interval) ANOVA. Greenhouse-Geisser adjusted degrees of freedom (Greenhouse \& Geisser, 1959) were used for the main effect and the interaction involving the lead interval factor. Post hoc comparisons examining differences between means were performed with $t$ tests. The critical values for these $t$ tests were derived from Sidak's tables, to protect against the accumulation of $\alpha$-error (Rohlf \& Sokal, 1981). The lead interval at which short lead interval inhibition was maximal was determined separately for each participant. Since the levels of the lead interval factor were not interval scaled, comparisons of the lead interval of maximal inhibition across groups were conducted with Mann-Whitney $U$ tests. The level of significance was set at .05 for all the analyses.

\section{Results}

The three groups did not differ in responsiveness to the presentations of the blink-elicitingstimulus alone (acoustic, $M=215.57 \mu \mathrm{V}, S D=118.0$; visual, $M=201.23 \mu \mathrm{V}, S D=$ 169.0 ; tactile, $M=180.98 \mu \mathrm{V}, S D=139.16 ; F<1)$. Figure 1 shows the blink magnitude modulation for each of the lead stimulus modality groups. The development of short lead interval inhibition appeared to occur earlier for the acoustic lead stimuli than for the visual and tactile lead stimuli. The acoustic lead stimulus also resulted in long lead interval facilitation but no facilitation at the very short lead intervals, whereas the opposite was found for the visual and tactile lead stimuli. An inspection of the 95\% confidence intervals in the acoustic lead stimulus group showed that significant short lead interval inhibition occurred at the 30-, 60-, and 120-msec lead intervals and that significant long lead interval facilitation occurred at the 4,500-msec lead interval. No significant facilitation was shown at the -30 - or 0 -msec lead interval. The pattern of blink modulation that emerged for the visual lead stimulus group was similar to that for the tactile lead stimulus group. In both groups, significant very short lead interval facilitation was found at the 0 - and 30-msec lead intervals and significant short lead interval inhibition occurred at the 120- and 240-msec lead intervals. Facilitation at the 4,500-msec lead interval was not significant.

A closer examination of the development of blink modulation across the lead intervals was provided by the statistical analyses. The factorial ANOVA resulted in a main effect for lead interval $\left[F(7,350)=22.74, M S_{\mathrm{e}}=1,149\right.$, $\varepsilon=.73, p<.001]$ and a group $\times$ lead interval interaction $\left[F(14,350)=8.78, M S_{\mathrm{e}}=1,149, \varepsilon=.73, p<.001\right]$ whereas the main effect for group was not significant $(F<1)$. Post hoc comparisons were used to compare blink modulation across groups separately for each lead inter-

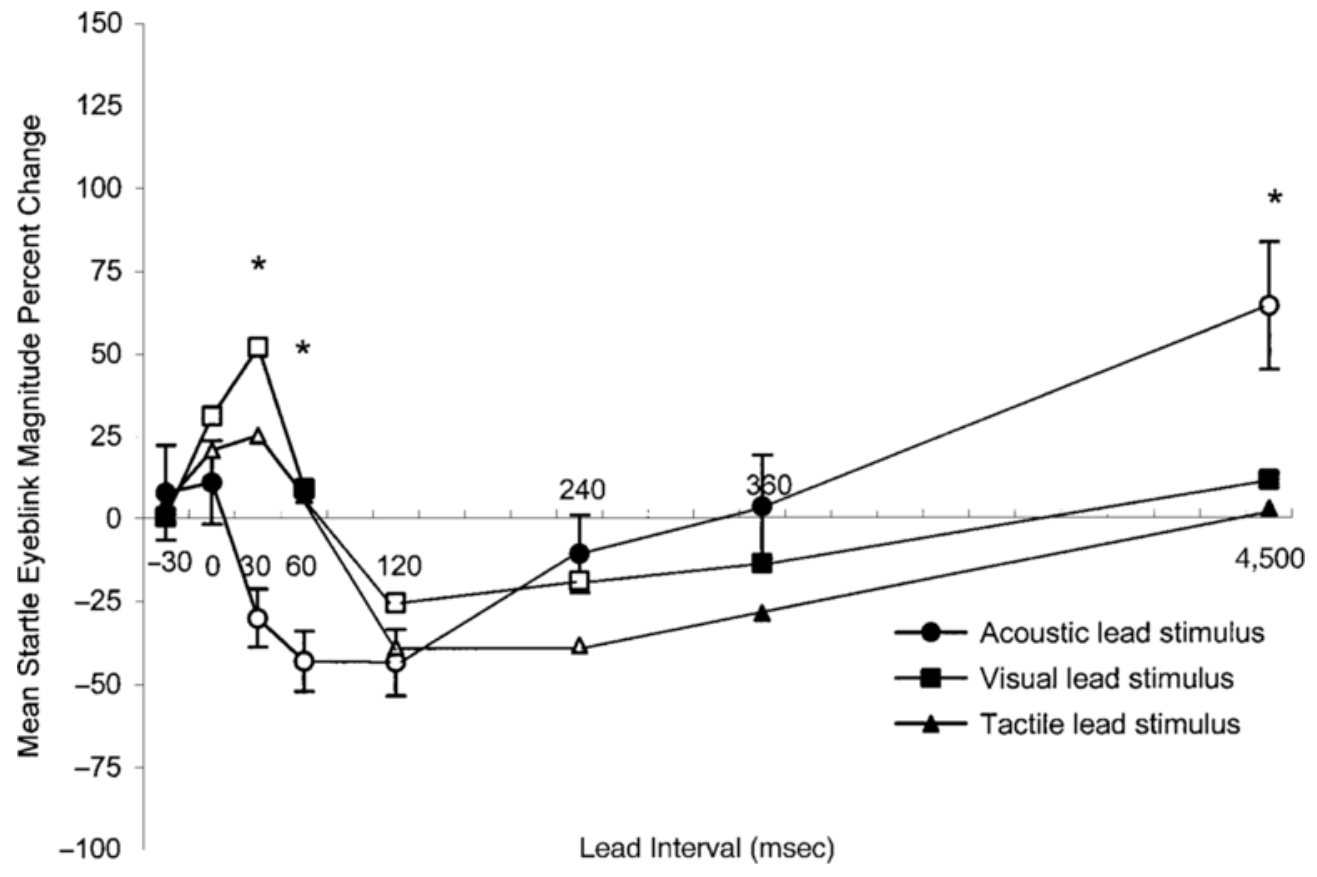

Figure 1. Percentage of change in mean blink reflex magnitude for an acoustic blink-eliciting stimulus at each lead interval in the acoustic, visual, and tactile lead stimulus groups in Experiment 1. Error bars indicate the standard errors of the means. Open symbols indicate that the mean was significantly different from baseline. Asterisks indicate a statistically significant difference between the acoustic lead stimulus group and both the visual and the tactile lead stimulus groups at that lead interval. 
val. The analyses showed that blink magnitude modulation differed between the acoustic and the visual lead stimulus groups at the $30-\mathrm{msec}[t(350)=7.28, p<.01]$ and the $60-\mathrm{msec}[t(350)=4.61, p<.01]$ lead intervals. Likewise, blink modulation differed between the acoustic and the tactile lead stimulus groups at the $30-\mathrm{msec}[t(350)=$ $4.91, p<.01]$ and the $60-\operatorname{msec}[t(350)=4.43, p<.01]$ lead intervals. Blink magnitude facilitation at the 4,500 msec lead interval was larger in the acoustic lead stimulus group than in the visual $[t(350)=4.67, p<.01]$ and the tactile $[t(350)=5.48, p<.01]$ lead stimulus groups. No other comparisons were significant (all $t \mathrm{~s}<2.44$ ).

The lead interval at which short lead interval inhibition was maximal appeared to be shorter in the acoustic lead stimulus group $(M=120 \mathrm{msec}, S D=73.48, M d n=$ $120 \mathrm{msec})$ than in the visual $(M=180 \mathrm{msec}, S D=92.04$, $M d n=120 \mathrm{msec})$ and tactile $(M=206 \mathrm{msec}, S D=$ $80.29, M d n=240 \mathrm{msec}$ ) lead stimulus groups. The planned comparisons confirmed this effect by showing a significant difference between the acoustic and the visual lead stimulus groups $(U=90.0, p<.05)$ and between the acoustic and the tactile lead stimulus groups $(U=63.50$, $p<.01)$. The difference between the visual and the tactile lead stimulus groups was not significant $(U=131$, $p>.05)$.

\section{Discussion}

The results of the present experiment demonstrate that the time course of acoustic blink modulation for acoustic lead stimuli differed from that for visual and tactile stimuli. Very short lead interval facilitation occurred with visual and tactile lead stimuli at the 0 - and 30-msec lead intervals but was not found for acoustic lead stimuli at any lead interval. Short lead interval inhibition was observed for all the lead stimulus modalities. However, the lead intervals at which inhibition occurred were shorter for acoustic lead stimuli $(30,60$, and $120 \mathrm{msec})$ than for the visual and tactile lead stimuli (120 and $240 \mathrm{msec}$ ). In addition, the lead interval of maximal inhibition was shorter for acoustic lead stimuli than for visual and tactile lead stimuli. Finally, long lead interval facilitation at the 4,500msec lead interval occurred for acoustic lead stimuli, but not for visual and tactile lead stimuli.

Very short lead interval facilitation emerged when the lead stimulus and the blink-eliciting stimulus were from different sensory modalities. Previous research in which an acoustic blink-eliciting stimulus has been used has reported blink facilitation at a $60-\mathrm{msec}$ lead interval (Aitken et al., 1999; Graham, 1980; Lipp et al., 2001) and a 50msec lead interval (Blumenthal \& Gescheider, 1987). The present experiment showed facilitation only at earlier lead intervals $(0$ and $30 \mathrm{msec})$. The earlier development of short lead interval facilitation in the present study may reflect differences in the nature of the lead stimuli that were used. In the present experiment, a neutral stimulus (a geometric shape) was presented repeatedly. In contrast, Aitken et al. (1999) and Lipp et al. (2001) used words depicting threat or no-threat themes and letters in standard or
Gothic font, respectively, and the word or the letter changed from trial to trial. It may be that the increased complexity of the stimuli or the increased novelty across trials helped to increase the attention allocated to these lead stimuli and resulted in a delayed occurrence of very short lead interval facilitation. Further aspects of Lipp et al.'s (2001) findings support this interpretation.Lipp et al. (2001) reported that very short lead interval facilitation was observed only in participants who were required to pay close attention to the lead stimulus by reporting the identity of the letter (vowel or consonant). No facilitation at the $60-\mathrm{msec}$ lead interval was observed in participants who were simply asked to view the letters.

Unlike very short lead interval facilitation, short lead interval inhibition was observed for all lead stimulus modalities in the present experiment. The lead interval of maximal inhibition, however, was shorter for acoustic lead stimuli than for visual and tactile lead stimuli. To our knowledge, the present study is the first to calculate the lead interval of maximal inhibition as an index of short lead interval inhibition in humans. Although short lead interval inhibition occurred earlier for acoustic lead stimuli than for visual and tactile lead stimuli, a question remains as to whether the extent of inhibition was also greater for acoustic lead stimuli. The statistical analyses showed a statistically significant difference between the stimulus modalities at the 60-msec lead interval. However, the differing time course of short lead interval inhibition may render such a comparison invalid. The $60-\mathrm{msec}$ lead interval appears to reflect the latter part of very short lead interval facilitation for the visual and tactile lead stimuli, whereas the same lead interval reflects the early part of short lead interval inhibition for acoustic lead stimuli.

A comparison of short lead interval inhibition across different lead stimuli may be valid only if it is made at comparable lead intervals. The lead interval of maximal inhibition is comparable between different lead stimuli, since this reflects the maximal protection of preattentive processing, according to Graham's (1975) model. Thus, supplementary analyses were conducted by comparing the percentage of change scores at the lead interval for which short lead interval inhibition was maximal. When averaged across participants, the mean percentage of magnitude change appeared to differ between the acoustic $(M=$ $-65.46 \%, S D=21.78)$, the visual $(M=-34.78 \%, S D=$ $55.35)$, and the tactile $(M=-55.35 \%, S D=32.18)$ groups. The difference between the groups, as assessed by a oneway ANOVA, was significant $\left[F(2,50)=6.77, M S_{\mathrm{e}}=\right.$ $635.52, p<.01]$. Post hoc comparisons showed that the maximal short lead interval inhibition was greater in the acoustic lead stimulus group than in the visual lead stimulus group $[t(50)=3.55, p<.01]$, whereas all other comparisons did not reach the preset level of significance using $\alpha$ protected values (all $t \mathrm{~s}<2.37$ ). The analyses suggest that not only did short lead interval inhibition occur earlier for acoustic lead stimuli than for visual lead stimuli, but also the extent of maximal short lead interval inhibition was greater for acoustic lead stimuli. 
Long lead interval facilitation was greater for acoustic lead stimuli than for visual or tactile lead stimuli, in agreement with the findings of Lipp et al. (2000b). Graham (1975) has proposed that long lead interval facilitation reflects two processes that summate: an attentional-orienting process caused by temporal uncertainty regarding the onset of the blink-eliciting stimulus and a general activation effect caused by the sustained portion of the lead stimulus. However, facilitation was observed only for the acoustic lead stimuli, and not for the visual and tactile lead stimuli. It would be expected that the three lead stimuli do not differ in the extent to which they elicit an attentional-orienting process. Thus, the lack of facilitation for tactile and visual lead stimuli may suggest that attentional orienting was not maintained throughout the entire lead interval of $4,500 \mathrm{msec}$. The facilitation for the acoustic lead stimulus may thus primarily reflect a sensory-specific general activation effect.

\section{EXPERIMENT 2}

The aim of Experiment 2 was to extend the results of Experiment 1 by using electrical stimulation of the trigeminal nerve as the blink-eliciting stimulus. Electrotactileelicited blinks produce three response components that differ in latency: R1 (8-12 msec), R2 (25-40 msec), and R3 (75-90 msec). Only the R2 response component was examined in Experiment 2, since this corresponds to the visible contraction of the orbicularis oculi muscles and is more comparable to the blink elicited by an acoustic blinkeliciting stimulus.

Very short lead interval facilitation of the R 2 component of the electrotactile blink reflex has been reported for an acoustic lead stimulus (Boelhouwer et al., 1991; Schmolesky et al., 1996). The R2 component has also been found to be facilitated when an acoustic lead stimulus was presented 50 msec after the blink-eliciting stimulus (Boelhouwer et al., 1991). Sarno et al. (1997) reported three experiments in which modulation of the electrotactile blink reflex for acoustic, visual, and tactile lead stimuli was examined. For acoustic lead stimuli, the R2 blink component was facilitated at very short negative lead intervals and for simultaneous presentations, whereas it was facilitated at very short positive lead intervals for visual lead stimuli. No facilitation was found at any lead interval for tactile lead stimuli. The findings suggest that short lead interval facilitation depends on the lead stimulus's being presented in a modality other than that of the blink-eliciting stimulus. However, definitive conclusions are limited by the fact that the stimuli used in the separate studies were not matched on their psychophysical intensity.

Short lead interval inhibition of the electrotactile blink reflex has also been observed for acoustic, visual, and tactile lead stimuli (Boelhouweret al., 1991; Sarno et al., 1997; Schmolesky et al., 1996). Short lead interval inhibition for an acoustic lead stimulus has been reported at lead intervals ranging from 50 to $120 \mathrm{msec}$ (Boelhouwer et al., 1991; Sarno et al., 1997; Schmolesky et al., 1996). Short lead interval inhibition for visual lead stimuli has been found at lead intervals of 60,80 , and $100 \mathrm{msec}$, although the specific lead interval at which inhibition was significant depended on the intensity of the lead stimulus (Sarno et al., 1997). Sarno et al. also reported significant inhibition at lead intervals of 60, 100, and $150 \mathrm{msec}$ for tactile lead stimuli. Like the very short lead interval effects, conclusions about the time course of short lead interval inhibition cannot be drawn from previous research, since the stimuli may have differed in psychophysical intensity and different lead intervals were used in each study. In addition, long lead interval facilitation of the electrotactile blink reflex has not been extensively examined in previous research.

Experiment 2 replicated the methodology in Experiment 1 in that the same lead intervals and similar lead stimuli were used, but the blink reflex was elicited by electrical stimulation of the trigeminal nerve. If very short lead interval facilitation is dependent on presentation of the lead stimulus and the blink-eliciting stimulus in different sensory modalities, facilitation would be expected to be found only for acoustic and visual lead stimuli. Likewise, a pattern of results different from that in Experiment 1 would be predicted if short lead interval inhibition and long lead interval facilitation is affected by the combination of the lead stimulus and the blink-eliciting stimulus modalities. Short lead interval inhibition and long lead interval facilitation would be predicted to be more pronounced for tactile lead stimuli than for visual and acoustic lead stimuli, due to the modality match of the tactile lead stimulus and the blink-eliciting stimulus.

\section{Method}

Participants. Twenty-one male and 45 female University of Queensland students who did not participate in Experiment 1 provided informed consent before participating. All the participants received course credit or AUS $\$ 10$ for their participation. The data from 4 male and 12 female participants were excluded due to excessive missing blink reflex data, and a further 2 male and 4 female participants were excluded due to extreme values in the blink reflex data. The final sample had a mean age of 19.2 years $(S D=2.7)$. The participants were randomly allocated to one of the three groups upon arrival at the laboratory in such a way that the final sample sizes and the male-to-female ratios were as follows: acoustic lead stimulus group, 5:9; visual lead stimulus group, 3:11; and tactile lead stimulus group, 7:9. The groups did not differ in sex distribution $\left[\chi^{2}(2)=\right.$ $1.39]$ or age $[F(3,42)=1.16]$.

Apparatus and Procedure. The apparatus and procedure were identical to those used in Experiment 1, with the exception of the equipment used to process the EMG signal, to present the visual lead stimulus, and to elicit the blink reflex. The raw EMG signal was amplified with a Coulbourn Instruments S75-01 bioamplifier, using a high-pass cutoff of $90 \mathrm{~Hz}$, a low-pass cutoff of $1000 \mathrm{~Hz}$, and a gain setting of 6000. The EMG signal was digitized and sampled on line, using a sampling rate of $1000 \mathrm{~Hz}$ in a time window of $100 \mathrm{msec}$ prior to onset and $400 \mathrm{msec}$ after offset of the blink-eliciting stimulus. The visual lead stimulus consisted of a white circle (RGB: 0, 0,0) centered on a dark gray background (RGB: 204, 204, 204). The stimuli were projected onto the projection area with a Compaq Contura 410 C computer, together with a Sony SVGA VPC-CS2 data projector and a Gerbrand Model G1166(S) tachistoscopic shutter.

As in Experiment 1, pilot work was conducted to ensure that the lead stimuli were matched on perceived intensity. The stimulation produced by the vibration unit was kept constant, and the intensities 
of the tone and the slide stimuli were varied. The participants received 5-sec presentation pairs of the vibration tone or vibration slide, separated by a 20 -sec interval. The participants were asked to indicate whether the intensity of the tone or the slide should be increased (or decreased) so that it matched that of the vibration. If the participant requested that the intensity of the tone or the slide be changed, the stimulus was varied, and it was presented with the vibration again. The intensity of the tone was varied in 5-dBA steps. The intensity of the slide was varied by changing the lightness of the gray background that was behind the white circle (high intensities) and by using a black background and varying the darkness of the gray in which the circle was drawn (low intensities). The procedure was repeated until the participants reported that the intensity of the tone or the slide matched the intensity of the vibration. The matching process was performed once in an upward and once in a downward staircase method.

The blink reflex was elicited by presenting an electric pulse with a Grass SD9 stimulator coupled in series with a Grass constant current unit. The $0.1-\mathrm{msec}$ electrical pulse was applied to the right supraorbital branch of the trigeminal nerve via two 4-mm-diameter $\mathrm{Ag} / \mathrm{AgCl}$ domed electrodes filled with a standard electrolyte (Surgicon E10). Electric pulse intensity was adjusted individually until it reliably elicited a blink reflex on repeated presentations. The mean intensities across participants was $11.0 \mathrm{~mA}(S D=3.7$, range $=$ 1-19 mA). The stimulus sequence, stimulus presentations, and intertrial intervals were controlled by a Dell OptiPlex 466/Le computer that also sampled the physiological signals.

Scoring, response definition, and statistical analysis. The response definition and scoring followed the methods used in Experiment 1 . The statistical analyses were similar to those in Experiment 1 . In addition, the short lead interval that produced the maximal inhibition was determined for each participant. The inhibition at that lead interval was averaged separately for each group, and group differences were examined with a one-way ANOVA.

\section{Results}

The mean blink magnitude on blink-eliciting stimulusalone trials did not differ across the acoustic $(M=$ $106.67 \mathrm{~A} / \mathrm{D}$ units, $S D=106.97)$, visual $(M=161.96 \mathrm{~A} / \mathrm{D}$ units, $S D=129.38)$, and tactile $(M=91.87 \mathrm{~A} / \mathrm{D}$ units, $S D=80.98$ ) lead stimulus groups (all $F \mathrm{~s}<1.76$ ). Blink magnitude modulation for the three groups at each lead interval is shown in Figure 2. In the acoustic lead stimulus group, significant facilitation was found at the -30 and $0-\mathrm{msec}$ lead intervals, significant inhibition emerged at the 60-, 120-, 240-, and 360-msec lead intervals, and significant long lead interval facilitation occurred at the 4,500-msec lead interval. In the visual and tactile lead stimulus groups, no facilitation at very short lead intervals was observed, and short lead interval inhibitionemerged at lead intervals of $60,120,240$, and $360 \mathrm{msec}$. No long lead interval facilitation was found at the 4,500-msec lead interval in either group.

The statistical analyses confirmed the effects of lead interval and lead stimulus modality by yielding a main effect for lead interval $\left[F(7,287)=48.68, M S_{\mathrm{e}}=2,108, \varepsilon=\right.$ $.63, p<.001]$ and a group $\times$ lead interval interaction $\left[F(14,287)=6.10, M S_{\mathrm{e}}=2,108, \varepsilon=.63, p<.001\right]$. The

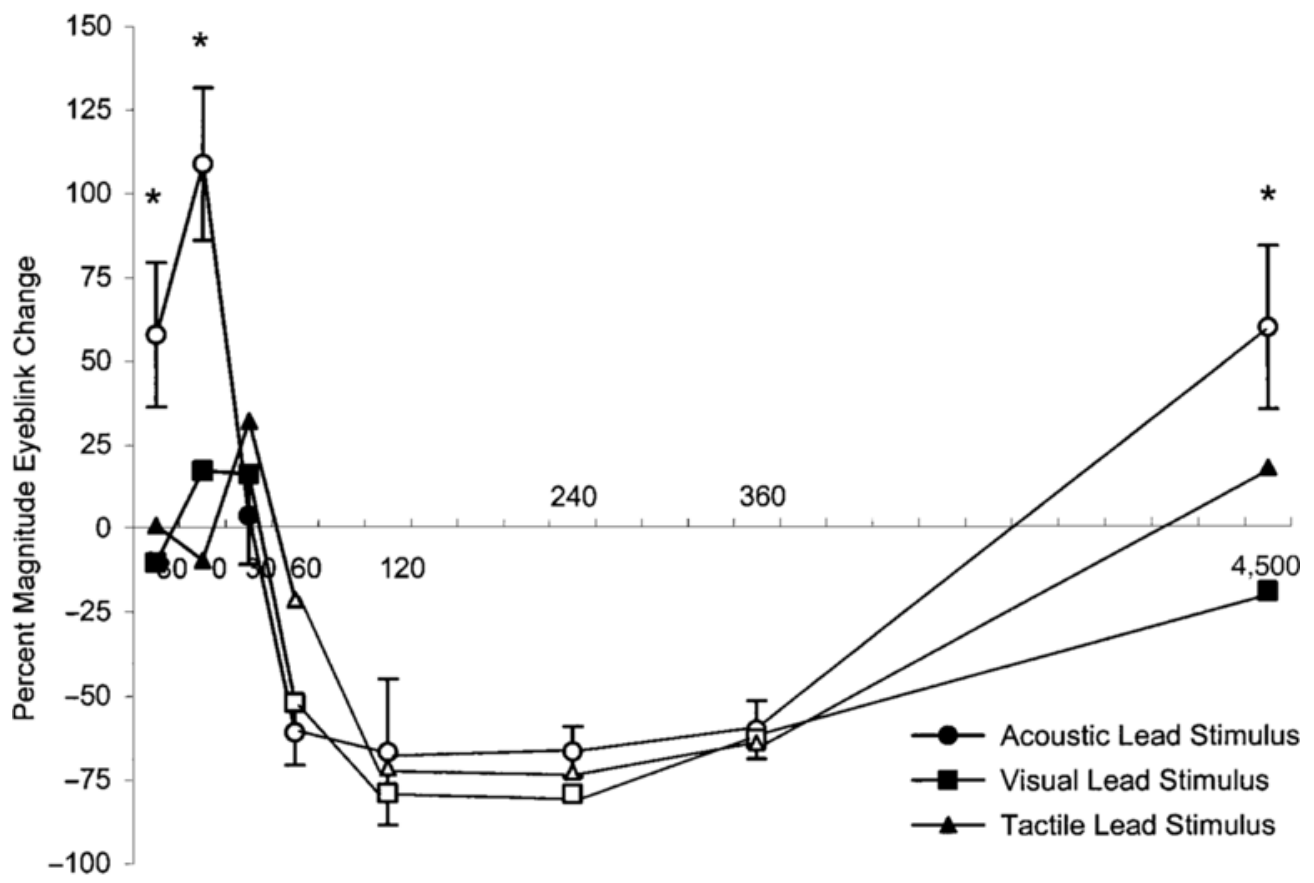

Lead Interval (msec)

Figure 2. Percentage of change in mean blink reflex magnitude for an electrotactile blink-eliciting stimulus at each lead interval in the acoustic, visual, and tactile lead stimulus groups in Experiment 2. Error bars indicate the standard errors of the means. Open symbols indicate that the mean was significantly different from baseline. Asterisks indicate a statistically significant difference between the acoustic lead stimulus group and the visual and tactile lead stimulus groups at the -30 - and 0 -msec lead intervals and a significant difference between the acoustic and the visual lead stimulus groups at the 4,500-msec lead interval. 
main effect for group did not reach the preset level of significance $\left[F(2,41)=2.91, M S_{\mathrm{e}}=5,870, p=.07\right]$. Post hoc comparisons between groups at each lead interval showed that facilitation at the -30 - and 0 -msec lead intervals was significantly greater in the acoustic lead stimulus group than in the visual lead stimulus group $[t(287)=4.67, p<$ .01 , and $t(287)=5.04, p<.01$, respectively $]$ and in the tactile lead stimulus group $[t(287)=4.19, p<.01$, and $t(287)=7.12, p<.01$, respectively]. Long lead interval facilitation at the 4,500-msec lead interval was significantly greater in the acoustic lead stimulus group than in the visual lead stimulus group $[t(287)=3.43]$, but not in the tactile lead stimulus group $[t(287)=1.52, p>.05]$. No other comparisons were significant (all $t \mathrm{~s}<2.65$ ).

The lead interval at which short lead interval inhibition was maximal appeared to differ between the acoustic $(M=$ $145.71 \mathrm{msec}, S D=80.55, M d n=120 \mathrm{msec}$ ), the visual $(M=231.43 \mathrm{msec}, S D=87.61, M d n=240 \mathrm{msec})$, and the tactile $(M=243.75 \mathrm{msec}, S D=103.85, M d n=240 \mathrm{msec})$ lead stimulus groups. The analyses confirmed this impression, since the lead interval of maximal inhibition was shorter in the acoustic lead stimulus group than in the visual $(U=45, p<.01)$ and tactile $(U=51.5, p<.01)$ lead stimulus groups. The visual and tactile lead stimulus groups did not differ $(U=105, p>.05)$. The extent of inhibition at the lead interval of maximal inhibition appeared to be the same for the acoustic $(M=-88.74 \%, S D=$ $10.32)$, the visual $(M=-83.70 \%, S D=14.09)$, and the tactile $(M=-87.77 \%, S D=14.86)$ lead stimulus groups. The statistical analyses did not yield a significant difference $(F<1)$.

\section{Discussion}

The results of the present experiment may be summarized as follows. Very short lead interval facilitation was observed at lead intervals of -30 and $0 \mathrm{msec}$ for acoustic lead stimuli, but not for visual and tactile lead stimuli. Short lead interval inhibition was observed at the same lead intervals for all lead stimulus modalities $(60,120,240$, and $360 \mathrm{msec}$ ), although the lead interval of maximal inhibition was shorter for acoustic lead stimuli than for visual and tactile lead stimuli. Long lead interval facilitation was observed for acoustic lead stimuli, but not for visual and tactile lead stimuli. The results of the present experiment show that the time course of electrotactile blink modulation for acoustic lead stimuli is different from that for visual and tactile lead stimuli. The time course of modulation of electrotactile blink for visual and tactile lead stimuli does not differ.

In agreement with previous research, very short lead interval facilitation was observed for an acoustic lead stimulus at a very short negative $(-30 \mathrm{msec})$ and a simultaneous $(0 \mathrm{msec})$ lead interval. Using white noise as a lead stimulus, Boelhouwer et al. (1991) reported significant facilitation at lead intervals ranging from -60 to $10 \mathrm{msec}$, and Sarno et al. (1997) reported facilitation at lead intervals ranging from -50 to $0 \mathrm{msec}$. Schmolesky et al. (1996) used a tone lead stimulus but found facilitation at different lead intervals than those reported in the present study and by Boelhouwer et al. and Sarno et al. Schmolesky et al. reported significant facilitation at lead intervals ranging from -20 to $40 \mathrm{msec}$. The finding of very short lead interval facilitation at longer lead intervals probably reflects differences in the rise time of the acoustic stimulus between the studies. In the present study and in Boelhouwer et al., the acoustic lead stimulus was presented with an instantaneous rise time, and a $5-\mathrm{msec}$ rise time was used by Sarno et al. In contrast, a $20-\mathrm{msec}$ rise time was used by Schmolesky et al. The delay in the peak intensity of the acoustic stimulus used by Schmolesky et al. appears to explain why significant facilitation was found at later lead intervals in their study.

In disagreement with the research reported by Sarno et al. (1997), no very short lead interval facilitation was found for a visual lead stimulus. The reason for this discrepancy is not clear. Sarno et al. used a light emitted from an incandescent light bulb at three different intensities and rise times as the lead stimulus, whereas in the present study a projected image with an instantaneous onset was used. Sarno et al. found that very short lead interval facilitation occurred at shorter lead intervals as the intensity and rise time of the visual stimulus were increased and shortened, respectively (for lights emitted by an incandescent bulb, both of these parameters vary together). It could be that the intensity or the instantaneous rise time of the stimuli used in the present experiment would have resulted in short lead interval facilitation if lead intervals earlier than $-30 \mathrm{msec}$ had been employed. However, the fact that the percentage of change of blink was smaller, although not significantly so, at the -30 -msec lead interval than at the 0 -msec lead interval argues against this possibility.

Short lead interval inhibition was observed with all the lead stimuli in the present study. This finding extends results from previous experiments in which short lead interval inhibition of electrotactile blinks was examined for lead stimuli that may have differed in psychophysical intensity (Boelhouweret al., 1991; Sarno et al., 1997; Schmolesky et al., 1996). In addition, in the present study, a wider range of lead intervals was used in order to better examine the development of short lead interval inhibition. An inspection of Figure 2 shows that the extent of short lead interval inhibition did not vary widely across the 120-, 240-, and 360-msec lead intervals for all the lead stimuli (cf. Figure 1). However, the calculation of the lead interval of maximal inhibition showed that maximal inhibition occurred earlier for acoustic lead stimuli than for visual and tactile lead stimuli. The finding of a difference between stimulus modalities provides further support that the calculation of the lead interval of maximal inhibition may offer a useful source of information. It provides a measure that is easily quantifiable and permits cross-modal comparisons that may highlight important differences of the temporal parameters in the convergence of sensory pathways that underlie short lead interval inhibition. The present study shows that the lead interval of maximal inhibition is sensitive to the sensory characteristics of the lead stimulus (stimulus modality). Future research may help 
determine whether it is also sensitive to other features of the lead stimulus and the blink-eliciting stimulus, such as intensity, rise time, or duration. Moreover, attentional processes have been shown to influence short lead interval inhibition, so that short lead interval inhibition is increased when a participant is required to pay attention to the stimulus (Lipp et al., 2001; see also the review by Filion et al., 1998). The lead interval of maximal inhibition may also differ depending on whether participants attend to or ignore the lead stimulus.

\section{GENERAL DISCUSSION}

The results of the present study indicate that the time course of blink reflex modulation is influenced by the modality of the lead stimulus and the modality of the blink-eliciting stimulus. In both experiments, the time course of blink modulation for the acoustic lead stimuli differed from that for the visual and tactile lead stimuli. The time course of blink modulation for the visual and the tactile lead stimuli did not differ. The presence of very short lead interval facilitation for a specific lead stimulus modality was dependent on the modality of the blink-eliciting stimulus. In contrast, the effect of lead stimulus modality on short lead interval and long lead interval modification effects were similar for both of the blink-eliciting stimuli used in the present experiments. Blink-eliciting stimulus modality therefore seems to be an important factor for very short lead interval modification, but not for short or long lead interval modification.

The notion that very short lead interval facilitation reflects temporal summation (Boelhouwer et al., 1991) suggests that the presence of short lead interval facilitation of blink may be useful in determining the convergence and conduction velocities within sensory pathways (Sarno et al., 1997). The present findings suggest that there is a convergence of the tactile and visual pathways with the acoustic pathway involved in the acoustic blink (Experiment 1) and a convergence between the acoustic pathway and the tactile pathway involved in the electrically elicited blink (Experiment 2). Contrary to previous reports (Sarno et al., 1997), there was no evidence of a convergence between the visual pathway and the tactile pathway involved in the electrically elicited blink reflex. The conduction velocities within the acoustic, visual, and tactile pathways differed, as was demonstrated by the lead intervals at which significant very short lead interval facilitation was observed. If one sensory pathway conducts information more rapidly than another, the simultaneous presentations of the lead stimulus and the blink-eliciting stimulus may not necessarily result in facilitation.

In both experiments, very short lead interval facilitation with acoustic and tactile (or electrotactile) stimuli occurred only when the tactile stimulus was presented simultaneously with or prior to the acoustic stimulus ( 0 - and 30-msec lead intervals in Experiment 1 and -30- and 0msec lead intervals in Experiment 2). This suggests that the conduction velocity within the acoustic pathway is up to $30 \mathrm{msec}$ faster than that in the tactile pathway. An inspection of Figure 1 suggests that the peak facilitation of acoustic blinks for tactile lead stimuli occurred at $30 \mathrm{msec}$. Figure 2 indicates that the peak facilitation of electrotactile blinks for acoustic lead stimuli occurred at $0 \mathrm{msec}$. The difference in the lead intervals may reflect, in part, the different locations of the stimuli in the two experiments, since the tactile stimulus was placed on the hand in Experiment 1, as compared with the forehead in Experiment 2 . The hand placement would be expected to induce a significant delay in the conduction of the signal to the neurological center at which temporal summation occurs, due to the need for the sensory impulse to travel up the arm. Bergenheim, Johansson, Granlund, and Pederson (1996) calculated that the difference in conduction time between tactile stimuli presented to the arm and the foot would be $24 \mathrm{msec}$, although the participants in their study reported the perception of simultaneous stimulus presentations if the tactile stimulus to the foot was presented $11.5 \mathrm{msec}$ before the tactile stimulus to the arm. In addition, the nature of the stimulation produced by the stimuli in the two experiments may more properly map onto different modality systems. The tactile lead stimulus would be expected to stimulate the vibrotactile system, whereas the electrotactile blink-eliciting stimulus would stimulate the trigeminal sensory system. It may also be inferred from the findings of Experiment 1 that the conduction velocity of the acoustic sensory pathway is up to $30 \mathrm{msec}$ faster than that in the visual pathway, since facilitation occurred at lead intervals of 0 and $30 \mathrm{msec}$.

Maximal short lead interval inhibition was found at a shorter lead interval for acoustic lead stimuli, relative to visual and tactile lead stimuli. One interpretation of this finding is that the timing of short lead interval inhibition is related to the process of very short lead interval facilitation. Short lead interval inhibition would thus be expected to be delayed whenever very short lead interval facilitation occurs if the neural mechanisms that underlie both processes act antagonistically at the level of sensory processing or motor output. This interpretation can account for the findings of Experiment 1, because the visual and tactile lead stimuli resulted in significant very short lead interval facilitation but in a delayed lead interval of maximal inhibition. However, it does not account for the findings of Experiment 2, because very short lead facilitation was found with acoustic lead stimuli and this stimulus modality also yielded a shorter lead interval of maximal inhibition. Thus, it appears that there is relatively minimal interference between the temporal summation process thought to underlie very short lead interval facilitation (Boelhouwer et al., 1991) and the protection of preattentive processing mechanism that is thought to underlie short lead interval inhibition (Graham, 1975).

The shorter lead interval of maximal inhibition for acoustic lead stimuli than for visual and tactile lead stimuli may reflect unique properties in the processing of acoustic stimuli. At a simple sensory level, it could reflect the overall conduction speed in the processing of acoustic 
information. As can be deduced from the time course of very short lead interval facilitation, sensory information is conducted faster in the acoustic pathway than in the visual and tactile pathways. The faster transmission of sensory information will mean that the processes that underlie short lead interval inhibition will be initiated at a shorter time following the onset of acoustic lead stimuli than following that of visual and tactile lead stimuli. Another interpretation, which may be complementary to this one, is that the neurological process that underlies short lead interval inhibition occurs early within the auditory system. In research with rats, Bellingham and Walmsley (1999) showed that there is a paired pulse synaptic depression in the cochlear nucleus, including an NMDA receptor-mediated paired pulse depression that occurs at intervals of 50$80 \mathrm{msec}$. This early time course of paired pulse depression indicates that there is a transient inhibitory process that occurs early in the auditory system. The early paired pulse depression, which appears to depend on a presynaptic mechanism, may thus contribute to the shorter lead interval of maximal inhibition for acoustic lead stimuli.

The effect of lead stimulus modality on long lead interval facilitation was independent of the modality of the blink-eliciting stimulus, in that facilitation was observed for acoustic lead stimuli in both experiments. No facilitation occurred for visual and tactile lead stimuli. These findings complement reports from studies in which neutral stimuli have been used in a passive attention task (Lipp et al., 2000b) or in an attention-demandingtask (Lipp et al., 2000a) with an acoustic blink-eliciting stimulus. The findings do not support the view that the direction of long lead interval blink-eliciting modulation is dependent on the modality match or mismatch between the lead stimulus and the blink-eliciting stimulus (Putnam, 1990). In both experiments, long lead interval facilitation for visual and tactile lead stimuli was not significantly different from baseline. According to Graham's (1975) model of long lead interval facilitation, it might have been expected that some facilitation would have been observed, due to an attentional-orienting process initiated by the temporal uncertainty regarding the onset of the blink-eliciting stimulus. The lack of facilitation indicates that the participants did not maintain orienting to the lead stimulus throughout the entire 4,500-msec lead interval. However, in the absence of an independent measure of attentional orienting, such as heart rate deceleration, it is difficult to substantiate this possibility. If there was a lack of attentional orienting to the lead stimulus presentation, the significant facilitation for acoustic lead stimuli appears primarily to reflect Graham's (1975) hypothesized general activation caused by the sustained portion of the lead stimulus. This interpretation would lead to the prediction that no facilitation will be observed for any lead stimulus modality with the present experimental design if discrete, rather than sustained, lead stimuli are used.

The lead stimuli used in the present experiments were equated in terms of their psychophysicalintensity. This al- lowed a comparison between lead stimuli from different stimulus modalities without any confound of differences in stimulus intensity. However, it is interesting to speculate about the degree to which the present modality effects were dependent on the level of intensity used for the lead stimuli. For instance, long lead interval facilitationincreases as the intensity of an acoustic lead stimulus is increased from 35 to $75 \mathrm{~dB}$ (Putnam, 1975; cited in Putnam \& Vanman, 1999). However, it is unlikely that intensity effects will be different for different lead stimulus modalities. Neumann and Lipp (2003) reported that long lead interval facilitation of the acoustic blink reflex was greater as the intensity of an acoustic and a visual lead stimulus was increased by the same level of psychophysical intensity. Short lead interval inhibition of the acoustic blink reflex is also greater as the intensity is increased for acoustic lead stimuli (e.g., Graham \& Murray, 1977) and tactile lead stimuli (Blumenthal \& Gescheider, 1987). Thus, it would seem that the modality effects reported in the present experiments would generalize across a relatively wide range of lead stimulus intensities. It is also interesting to note that the intensity of the blink-eliciting stimulus may be a second, possibly relevant factor in the effects of stimulus modality on blink modulation. The approach adopted in the present experiment was to use different lead stimulus modalities matched on psychophysical intensity and one blink-eliciting stimulus within one experiment. An equally valid approach could be to use one lead stimulus modality and different blink-eliciting stimulus modalities matched on psychophysical intensity.

The aim of the present study was to provide the first systematic investigation of the effects of lead stimulus and blink-eliciting stimulus modality on blink modulation across a wide range of lead intervals. The wide range of lead intervals enabled the simultaneous examination of very short, short, and long lead interval effects. Future research can expand upon the present findings in a number of ways. For instance, a novel approach in the present experiment was to examine what lead interval produced maximal short lead interval inhibition. An even finer grained analysis of the lead interval of maximal inhibition could be achieved by using a larger number of lead intervals. The present study highlighted important differences in the time course of blink modulation for the different combinations of lead stimulus and blink-eliciting stimulus modalities when sustained lead stimuli were used. The present findings can be extended to parallel studies that use discrete lead stimuli to highlight the separate contributions that the sustained portion of the lead stimulus has in producing blink modulation, particularly at long lead intervals. The present research adds to an accumulating body of evidence (e.g., Lipp et al., 2000a, 2000b; Putnam, 1990; Rimpel et al., 1982; Sarno et al., 1997) that stimulus modality is an important factor in the modulation of blink reflexes and, as a consequence, to our understanding of those psychological processes that are thought to be reflected in blink reflex modulation. 


\section{REFERENCES}

Aitken, C. J., Siddle, D. A. T., \& Lipp, O. V. (1999). The effects of threat and non-threat word lead stimuli on blink modification. Psychophysiology, 36, 699-705.

Bellingham, M. C., \& Walmsley, B. (1999). A novel presynaptic inhibitory mechanism underlies pair pulse depression at a fast central synapse. Neuron, 23, 159-170.

BERG, W. K., \& BALABAN, M. T. (1999). Startle elicitation: Stimulus parameters, recording techniques, and quantification. In M. E. Dawson, A. M. Schell, \& A. H. Böhmelt (Eds.), Startle modification: Implications for neuroscience, cognitive science, and clinical science (pp. 2150). New York: Cambridge University Press.

Bergenheim, M., Johansson, H., Granlund, B., \& Pederson, J. (1996). Experimental evidence for a synchronization of sensory information to conscious experience. In S. R. Hameroff, A. W. Kaszniak, \& A. C. Scott (Eds.), Toward a science of consciousness: The first Tucson discussions and debates (pp. 303-310). Cambridge, MA: MIT Press.

Blumenthal, T. D. (1999). Short lead interval startle modification. In M. E. Dawson, A. M. Schell, \& A. H. Böhmelt (Eds.), Startle modification: Implications for neuroscience, cognitive science, and clinical science (pp. 51-71). New York: Cambridge University Press.

Blumenthal, T. D., \& Gescheider, G. A. (1987). Modification of the acoustic startle reflex by a tactile prepulse: The effects of stimulus onset asynchrony and prepulse intensity. Psychophysiology, 24, 320327.

Blumenthal, T. D., \& Tolomeo, E. A. (1989). Bidirectional influences of vibrotactile stimuli on modification of the human acoustic startle reflex. Psychobiology, 17, 315-322.

Boelhouwer, A. J. W., Teurlings, R. J., \& Brunia, C. H. (1991). The effect of an acoustic warning stimulus upon the electrically elicited blink reflex in humans. Psychophysiology, 28, 133-139.

Dell'Acqua, R., Turatto, M., \& JolicÄUr, P. (2001). Cross-modal attentional deficits in processing tactile stimulation. Perception \& Psychophysics, 63, 777-789.

DRIVER, J., \& SPENCE, C. (2001). Cross-modal links in spatial attention. In G. W. Humphreys, J. Duncan, \& A. Treisman (Eds.), Attention, space, and action: Studies in cognitive neuroscience (pp. 130-149). New York: Oxford University Press.

Filion, D. L., DAwson, M. E., \& SCHell, A. M. (1998). The psychological significance of human startle eyeblink modification: A review. Biological Psychology, 47, 1-43.

Flaten, M. A., \& Blumenthal, T. D. (1996). Effects of positive and negative stimulus onset asynchronies between weak stimuli and blink reflex eliciting stimuli. Journal of Psychophysiology, 10, 189-197.

GRAHAM, F. K. (1975). The more or less startling effects of weak prestimulation. Psychophysiology, 12, 238-248.

Graham, F. K. (1980). Control of blink reflex excitability. In R. F. Thompson, L. H. Hicks, \& V. B. Shvyrkov (Eds.), Neural mechanisms of goal-directed behavior and learning (pp. 511-519). New York: Academic Press.

Graham, F. K., \& Murray, G. M. (1977). Discordant effects of weak pre-stimulation on magnitude and latency of the reflex blink. Physiological Psychology, 5, 108-114.
Graham, F. K., Putnam, L. E., \& Leavitt, L. A. (1975). Lead stimulation effects on human cardiac orienting and blink reflexes. Journal of Experimental Psychology: Human Perception \& Performance, 104, 161-169.

Greenhouse, S. W., \& Geisser, S. (1959). On methods in the analysis of profile data. Psychometrika, 24, 95-112.

Lipp, O. V., Blumenthal, T. D., \& Adam, A. R. (2001). Attentional modulation of blink startle at long, short, and very short lead intervals. Biological Psychology, 58, 89-103.

LiPP, O. V., SidDLE, D. A. T., \& DALL, P. J. (2000a). The effect of warning stimulus modality on blink startle modulation in reaction time tasks. Psychophysiology, 37, 55-64.

LipP, O. V., Siddle, D. A. T., \& Dall, P. J. (2000b). The effects of change in lead stimulus modality on the modulation of acoustic blink startle. Psychophysiology, 37, 715-723.

Neumann, D. L., \& LipP, O. V. (2003). The independent effects of attention and lead stimulus properties on the acoustic blink reflex. Journal of Psychophysiology, 17, 124-134.

PutNAM, L. E. (1990). Great expectations: Anticipatory responses of the heart and brain. In R. Johnson, J. W. Rohrbaugh, R. Parasuraman, \& R. Johnson, Jr. (Eds.), Event-related brain potentials: Basic issues and applications (pp. 178-209). New York: Oxford University Press.

Putnam, L. E., \& VANMAN, E. J. (1999). Long lead interval startle modification. In M. E. Dawson, A. M. Schell, \& A. H. Böhmelt (Eds.), Startle modification: Implications for neuroscience, cognitive science, and clinical science (pp. 72-92). New York: Cambridge University Press.

Rimpel, J., Geyer, D., \& Hopf, H. C. (1982). Changes in the blink responses to combined trigeminal, acoustic, and visual repetitive stimulation, studied in the human subject. Electroencephalography \& Clinical Neurophysiology, 54, 552-560.

Rohlf, F. J., \& SoKal, R. R. (1981). Statistical tables (2nd ed.). New York: Freeman.

Sarno, A. J., Blumenthal, T. D., \& Boelhoumer, A. J. W. (1997). Modification of the electrically elicited eyeblink by acoustic, visual, and vibrotactile pulses. Psychobiology, 25, 253-265.

Schmolesky, M. T., Boelhouwer, A. J. W., \& Blumenthal, T. D. (1996). The effect of acoustic pulse intensity upon the electrically elicited blink reflex at positive and negative stimulus onset asynchronies. Biological Psychology, 44, 69-84.

TE KOK-KILIC, A., SHUCARD, J. L., \& SHUCARD, D. W. (2001). Stimulus modality and go/no-go effects on P3 during parallel visual and auditory continuous performance tasks. Psychophysiology, 38, 578-589.

\section{NOTE}

1. The grouping of lead intervals as very short, short, and long is arbitrary and is defined by the time between the lead stimulus and the blink-eliciting stimulus onsets. There may be some overlap between the lead intervals used in each group that yield findings described as very short, short, and long lead interval effects due to particular features of the experimental design and stimuli used.

(Manuscript received January 14, 2002; revision accepted for publication June 4, 2003.) 